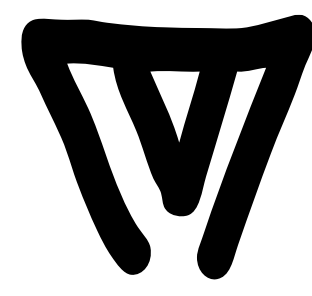

REVISTA DE ESTUDIOS E INVESTIGACIÓN

EN PSICOLOGÍA Y EDUCACIÓN

ISSN: 1138-1663; elSSN: 2386-7418

UDC / UMinho

2019, Vol. 6, No. 1, 37-43.

DOI: https://doi.org/10.17979/reipe.2019.6.1.5271

\title{
Diferencias en imagen mental entre individuos españoles e italianos
}

\author{
Differences in mental imagery between Spanish and Italian populations
}

\author{
Alfredo Campos (iD, María Canto \\ Universidad de Santiago de Compostela
}

\begin{abstract}
Resumen
Son pocos los estudios que miden la viveza de imagen entre individuos de diferentes países, por eso, en nuestra investigación deseábamos averiguar si existían diferencias significativas entre los individuos de España y de Italia, y entre las mujeres y hombres, en tres tipos de imágenes mentales: viveza de imagen, control de imagen, y habilidad para la imagen espacial. Para ello, seleccionamos un grupo de 103 estudiantes universitarios italianos y otro grupo de 110 estudiantes universitarios españoles a los que se les aplicaron tres tipos de test: un test que medía viveza de imagen, otro que medía el control de imagen, y el tercero que medía la habilidad de imagen espacial. Se encontró que el género no influía en ninguna de las medidas de imagen utilizada, sin embargo, el país influyó en el control de imagen. Los españoles obtuvieron puntuaciones, en control de imagen, significativamente superiores a las que obtuvieron los individuos italianos. Se necesitan nuevos estudios que midan estos y otros tipos de imagen, en estos y en otros países, para llegar a tener un conocimiento de cómo se distribuye la capacidad de imagen por todo el mundo.
\end{abstract}

Palabras clave: diferencias culturales, diferencias de género, imagen, control, imagen espacial

\begin{abstract}
Studies measuring imagery vividness in different populations from different countries are scarce. Thus, the aim of this study was to ascertain if there were significant differences between Spanish and Italian populations and between women and men in three types of mental imagery: image vividness, image control, and spatial imagery ability. A total of 103 Italian second-year undergraduates, and 110 Spanish second-year undergraduates were administered three types of test: a test measuring image vividness, another image control, and a third test measuring spatial imagery ability. Though gender was not found to influence any of the measurements of imagery analysed, the country of origin was found to influence image control. Spanish participants obtained significantly higher image control scores than Italian participants. Further studies are required to measure these and other types of imagery, in these and other countries in order to broaden our understanding of the distribution of imagery ability worldwide.
\end{abstract}

Keywords: cultural differences, gender differences, imagery, control, spatial imagery

\footnotetext{
Alfredo Campos (DD y María Canto: Departamento de Psicología Social, Básica y Metodologia. Rúa Xosé María Suárez Núñez, s/n. Campus Vida. 15782 Santiago de Compostela. España.

Correspondencia relativa a este artículo: Alfredo Campos-alfredo.campos@usc.es
} 


\section{Diferencias en imagen mental entre países}

Resulta sorprendente la cantidad de investigaciones que se han efectuado sobre la imagen mental, sin embargo, son muy pocas las investigaciones que se han hecho sobre la diferencia en imagen mental entre diferentes países (Marsella y Quijano, 1974). Baranchok (1995) efectuó una comparación de la viveza de imagen entre estudiantes universitarios de México y Estados Unidos. La medida utilizada fue el Betts' Questionnaire Upon Mental Imagery (Betts' QMI, Sheehan, 1967). Baranchok encontró que los estudiantes de México superaban a los de Estados Unidos en la viveza de imagen en las siete modalidades sensoriales de las que consta el test.

Marsella y Quijano (1974) utilizaron un grupo de 30 estudiantes universitarios filipinos y 30 americanos caucásicos descendientes de anglo-americanos, a los que les aplicaron el Betts' QMI (Sheehan, 1967). Las puntuaciones de los estudiantes filipinos fueron significativamente superiores a las de los estudiantes norteamericanos en las modalidades: cinestésica, gustativa, cutánea y olfativa. No encontraron diferencias significativas en la modalidad visual, auditiva y orgánica.

Otra forma de estudiar la diferencia entre las imágenes mentales de las personas de diferentes países, distinta a la de los test, es midiendo la viveza de imagen que suscitan las mismas palabras en distintos países (Campos y Astorga, 1986a, 1986b, 1986c). Campos y Astorga (1986a) utilizaron las 338 palabras que Walker (1970) había utilizado con individuos norteamericanos, y también utilizaron 111 palabras que habían utilizado Paivio, Yuille y Madigan (1968) en Canadá y que coincidían con las de Walker. Cada palabra fue valorada en viveza de imagen por 100 individuos, en una escala de 1 a 7 puntos. Campos y Astorga (1986a) encontraron una correlación de .93 entre la viveza de imagen de las mujeres y los hombres españoles. La puntuación en viveza de imagen de los estudiantes españoles correlacionó .88 con la de los estudiantes de Estados Unidos, y .88 con los estudiantes de Canadá. Al analizar la diferencia de las puntuaciones de imagen entre los estudiantes de los diferentes países, se encontró que los estudiantes españoles dieron puntuaciones significativamente inferiores a los canadienses (Campos y Astorga (1986b), sin embargo, dieron puntuaciones significativamente superiores a los estudiantes de

Estados Unidos (Campos y Astorga, 1986c). Estos resultados nos indican que pueden existir diferencias en imagen entre los individuos de diferentes países.

\section{Diferencias en imagen y género}

Muchos estudios han examinado las diferencias de género en la capacidad para formar imágenes mentales, pero los resultados encontrados siguen siendo inconsistentes, debido, fundamentalmente, a la abundancia de tipos de imágenes (Campos, 1998a, 2014; Horowitz, 1983, Richardson, 1977) y la disparidad entre las pruebas, cuya intención es medir la misma capacidad de formar imágenes mentales, sin embargo, fracasan al mostrar correlaciones entre ellas (Campos, 1998b, 2009, 2012).

Algunos estudios que analizaron las diferencias de género con cuestionarios de imagen encontraron que las mujeres manifestaban una mayor viveza de imagen que los hombres (Campos y Pérez, 1988a; Campos y Sueiro, 1993; McKelvie, 1986; White, Ashton, y Brown, 1977). En cambio, en otros estudios no se ha detectado diferencias significativas (Ashton y White, 1980; Campos, González, y Amor, 2002; Campos y Pérez, 1988b; McConkey y Nogrady, 1986). Más recientemente, algunas investigaciones utilizaron el Vividness of Visual Imagery Questionnaire-2 (VVIQ-2, Marks, 1995), que es la última versión del Vividness of Visual Imagery Questionnaire (Marks, 1973) y tampoco encontraron diferencias entre las mujeres y los hombres (Campos, 2014).

Campos, Pérez-Fabello y Gómez-Juncal (2004) no encontraron diferencias significativas entre las mujeres y los hombres en el control de imagen, en un grupo de mujeres y hombres de diferentes edades. Pérez-Fabello y Campos (2004) tampoco encontraron diferencias significativas entre las mujeres y los hombres en las puntaciones de control de imagen, en un grupo de 479 estudiantes universitarios. Campos $(2009,2012,2013)$ distingue entre habilidad de imagen espacial y habilidad para rotar imágenes mentales. En esta investigación se midió la diferencia 
entre mujeres y hombres en la habilidad para utilizar imágenes espaciales. Campos $(2009$, 2013) no encontró diferencias significativas entre las mujeres y los hombres en las habilidades para la imagen espacial. Linn y Peterson (1985), y Voyer, Voyer y Bryden (1995), en una meta-análisis encontraron diferencias significativas entre las mujeres y los hombres en rotación mental, pero no en percepción espacial. A la misma conclusión llegaron Reilly y Neumann (2013).

Probablemente, cuando se hable de diferencias de imagen en función del género de los participantes, y en función de los países en los que viven, habrá que especificar qué tipo de imagen se está midiendo y qué tipo de instrumento se utilizó en la medición (Campos et al., 2004). En nuestra investigación deseábamos averiguar si existía diferencia significativa entre mujeres y hombres, y los entre individuos de España y de Italia, en tres tipos de imágenes mentales: viveza de imagen, control de imagen, y habilidad para la imagen espacial.

\section{Método}

\section{Participantes}

Un grupo de 213 personas (103 italianos y 110 españoles; de los cuales 150 eran mujeres y 63 hombres), del segundo curso de la facultad de psicología de Milán (Italia) y de Santiago de Compostela (España), con una media de edad de 20.49 años $(S D=2.77)$, participó en este estudio. Todos ellos participaron de manera voluntaria en el estudio y se les garantizó la confidencialidad de los resultados.

\section{Instrumentos}

Se utilizó la versión española (Pérez-Fabello y Campos, 2004) del Gordon Test of Visual Imagery Control (GTVIC; Richardson, 1969). Este test mide la capacidad de control de las imágenes visuales que poseen los participantes, y está compuesto por 12 ítems en los que a los individuos se les pide que imaginen un coche en varios colores, posiciones y estados de movimiento. A continuación, deben evaluar cada ítem en una escala de 3 puntos, donde "no" se puntúa 0, "inseguro" se puntúa 1 y "sí" se puntúa 2. La puntuación total tiene un rango de 0 a 24 puntos. Pérez-Fabello y Campos (2004) obtuvieron un alfa de Cronbach de .69. La versión italiana fue elaborada por nosotros siguiendo la normativa internacional de traducción de test.

También se empleó la versión española de Campos et al. (2002) del Vividness of Visual Imagery Questionnaire (VVIQ; Marks, 1973). El test consta de 16 ítems que se contestan de dos formas, una con los ojos abiertos y, la segunda vez, con ellos cerrados. El participante evalúa la viveza de ciertos aspectos de la imagen que se valoran en una escala de 5 puntos, donde 5 es "Ninguna imagen, tú solo "sabes" lo que estás pensando del objeto" y el 1 es "Perfectamente clara y tan viva como si estuvieses viendo el objeto". Esto quiere decir que una puntuación baja señala una alta viveza de imagen, y una puntuación alta indica una baja viveza. Campos et al. (2002) obtuvieron un alfa de Cronbach de .88. La versión italiana fue elaborada por los autores del presente estudio, siguiendo la normativa de traducción de los test.

Además, se ha empleado la versión italiana (Varela, 2018) del Measure of the Ability to Form Spatial Mental Imagery (MASMI; Campos, 2009, 2013), y la versión española, que es la original del test (Campos, 2009, 2013). El test está formado por un cubo descompuesto que los participantes tienen que cerrar mentalmente para poder contestar a cada una de las 23 preguntas del test. Cada pregunta tiene cuatro respuestas, de las cuales dos son siempre ciertas y dos son siempre falsas. El total de la puntuación se consigue sumando las respuestas correctas y restando las respuestas incorrectas, por lo que la puntuación total en el test puede variar entre 46 y -46 . Los individuos tienen 5 minutos para responder a todas las preguntas del test. Campos (2009) obtuvo un alfa de Cronbach de .93 . 


\section{Procedimiento}

Para la versión italiana del VVIQ, en primer lugar, se tomó la versión inglesa y se tradujo al italiano. Posteriormente, una persona ajena a la investigación tradujo, de nuevo, el test al inglés para ver si eran iguales, y después entre los autores y la traductora, se volvió a traducir al italiano. Con la versión italiana del Gordon Test of Imagery Control se siguió el mismo procedimiento.

A los participantes, en sus clases habituales, en grupos de aproximadamente 20 estudiantes, se les pasaron las versiones españolas (Campos et al., 2002) del Vividness of Visual Imagery Questionnaire (VVIQ; Marks, 1973), la versión española (Pérez-Fabello y Campos, 2004) del Gordon Test of Visual Imagery Control (GTVIC; Richardson, 1969), y la versión española de la Measure of the Ability to Form Spatial Mental Imagery (MASMI; Campos, 2009, 2013). Se les entregó una carpetilla con los tres test y se les leyeron las instrucciones. Los test no tenían límite de tiempo, excepto el MASMI, para el que disponían de 5 minutos. Las pruebas se presentaron al azar, los estudiantes participaron voluntariamente, y se les garantizó la confidencialidad de los resultados. Con los participantes italianos se siguió el mismo procedimiento para pasar los test.

Se controlaron las siguientes variables para que los grupos fuesen lo más homogéneos posibles: los participantes eran todos de la enseñanza pública, del segundo curso de la facultad de psicología, tanto de Italia como de España, las pruebas se pasaron al azar a todos los grupos, las instrucciones y el tiempo fueron los mismos para todos los grupos, todos los individuos participaron voluntariamente, no tenían que poner su nombre ni nada que pudiese identificarlos, de este modo se intentaba que fuesen sinceros.

\section{Análisis de Datos}

Los análisis estadísticos se efectuaron con el SPSS 24. En primer lugar, se hizo un alfa de Cronbach para averiguar la consistencia interna de cada uno de los test, tanto de la versión española como italiana: VVIQ, Gordon Test y MASMI. En segundo lugar, para verficar si existían diferencia significativa entre las mujeres y los hombres, y entre los individuos de Italia y los de España en la viveza de imagen visual, se realizó un ANOVA de 2 (género) x 2 (país). La variable dependiente fue la viveza de imagen, medida por el VVIQ. Para averiguar si existía diferencia significativa entre las mujeres y los hombres, y entre los individuos de Italia y los de España en el control de imagen, se hizo un ANOVA de 2 (género) x 2 (país). La variable dependiente fue el control de imagen (Gordon Test). Finalmente, para averiguar si existía diferencia significativa entre las mujeres y los hombres, y entre los individuos de Italia y los de España en la habilidad de imagen espacial, se llevó a cabo un ANOVA de 2 (género) x 2 (país). La variable dependiente fue la habilidad de imagen espacial (MASMI).

\section{Resultados}

Para medir la consistencia interna del Vividness of Visual Imagery Questionnaire (VVIQ) se efectuó un alfa de Cronbach, y se encontró un alfa de .87 para la versión española, y un alfa de .90 para la versión italiana. Con el Gordon Test se obtuvo un alfa de Cronbach de .58 para la versión española, y un alfa de .71 para la versión italiana. Y, finalmente, se logró un alfa de Cronbach de .87 para la versión española, y de .84 para la versión italiana del MASMI.

Para verficar si existían diferencias significativas entre las mujeres y los hombres, y entre los individuos de Italia y los de España en la viveza de imagen visual, efectuamos un ANOVA de 2 (género) x 2 (país). La variable dependiente fue la viveza de imagen, medida por el VVIQ. Las medias y desviaciones típicas de cada grupo se encuentran en la Tabla 1. Los resultados del ANOVA indicaron que el género no influyó en la viveza de imagen, $F(1,209)=1.08, p=.30, \eta^{2}=.01$, potencia $=.18$. El país tampoco influyó en la viveza de imagen, $F(1,209)=$ $2.23, p=.14, \eta_{p}^{2}=.01$, potencia $=.32$. La interacción entre género y país tampoco fue significativa $F(1,209)=$ $3.00, p=.09, \eta^{2}=.01$, potencia $=.41$. 
Tabla 1

Medias y Desviaciones Típicas en Viveza de Imagen Visual (VVIQ)

\begin{tabular}{|c|c|c|c|c|c|c|}
\hline \multirow[b]{3}{*}{ Género } & \multicolumn{4}{|c|}{ País } & \multirow{2}{*}{\multicolumn{2}{|c|}{ Total }} \\
\hline & \multicolumn{2}{|c|}{ Italia } & \multicolumn{2}{|c|}{ España } & & \\
\hline & $M$ & $S D$ & $M$ & $S D$ & $M$ & $S D$ \\
\hline Mujeres & 2.42 & 0.62 & 2.11 & 0.45 & 2.26 & 0.56 \\
\hline Hombres & 2.16 & 0.66 & 2.18 & 0.62 & 2.17 & 0.63 \\
\hline Total & 2.37 & 0.64 & 2.13 & 0.49 & 2.24 & 0.58 \\
\hline
\end{tabular}

Nota: Altas puntuaciones en el VVIQ indican baja viveza de imagen, y viceversa.

También interesaba averiguar si había diferencias significativas en el control de imagen entre las mujeres y los hombres, y entre los estudiantes españoles e italianos. Las medias y desviaciones obtenidas por cada grupo se encuentran en la Tabla 2. El ANOVA resultante indicó que el género no influyó en el control de imagen $F(1,209)=1.03, p=$ $.31, \eta_{p}^{2}=.01$, potencia $=.17$; en cambio, el país sí influyó de manera significativa, $F(1,209)=4.32, p$ $=.04, \eta_{p}^{2}=.02$, potencia $=.54$. Los españoles obtuvieron puntuaciones superiores $(M=20.86, S D$ $=2.73$ ) a las puntuaciones obtenidas por los italianos $(M=19.19, S D=3.91)$. Por otra parte, la interacción entre género y país tampoco fue significativa, $F(1$, 209) $=1.92, p=.17, \eta_{p}^{2}=.01$, potencia $=.28$.

Finalmente, se quería averiguar si había diferencias significativas en imagen espacial (MASMI) entre las mujeres y los hombres, y entre los individuos de España e Italia. Las medias y desviaciones típicas de los distintos grupos se encuentran en la Tabla 3. El ANOVA indicó que el género no influyó en la habilidad espacial, $F(1,209)$ $=0.38, p=.54, \eta^{2}{ }_{p}=.01$, potencia $=.10$. De la misma manera, se encontró que el país tampoco influyó de manera significativa, $F(1,209)=0.17, p=.69, \eta_{p}^{2}$ $=.01$, potencia $=.07$. La interacción entre el género y el país tampoco fue significativa, $F(1,209)=0.29$, $p=.59, \eta_{p}^{2}=.01$, potencia $=.08$.

Tabla 2

Medias y Desviaciones Típicas del Control de Imagen (Gordon Test)

\begin{tabular}{|c|c|c|c|c|c|c|}
\hline \multirow[b]{3}{*}{ Género } & \multicolumn{4}{|c|}{ País } & \multirow{2}{*}{\multicolumn{2}{|c|}{ Total }} \\
\hline & \multicolumn{2}{|c|}{ Italia } & \multicolumn{2}{|c|}{ España } & & \\
\hline & $M$ & $S D$ & $M$ & $S D$ & $M$ & $S D$ \\
\hline Mujeres & 18.93 & 3.93 & 20.91 & 2.69 & 19.94 & 3.49 \\
\hline Hombres & 20.30 & 3.69 & 20.70 & 2.91 & 20.51 & 3.26 \\
\hline Total & 19.19 & 3.91 & 20.86 & 2.73 & 20.06 & 3.44 \\
\hline
\end{tabular}

Tabla 3

Medias y Desviaciones Típicas de Imagen Espacial (MASMI)

\begin{tabular}{lcccccc}
\hline & \multicolumn{4}{c}{ País } & \multicolumn{2}{c}{ Total } \\
\cline { 2 - 6 } Género & \multicolumn{2}{c}{ Italia } & \multicolumn{2}{c}{ España } & & \multicolumn{2}{c}{$S$} & $S D$ & $M$ & $S D$ & $M$ & $S D$ \\
\hline Mujeres & 9.67 & 8.89 & 8.24 & 8.15 & 8.94 & 8.53 \\
Hombres & 9.80 & 7.60 & 10.00 & 12.10 & 9.91 & 10.14 \\
Total & 9.70 & 8.62 & 8.61 & 9.08 & 9.14 & 8.86 \\
\hline
\end{tabular}

\section{Discusión}

La consistencia de los test se midió a través del alfa de Cronbach. George y Mallery (2003) afirman que, para este tipo de test, una puntuación superior a .60 es cuestionable, superior a .70 es aceptable, y superior a .80 es una puntuación buena. En función de esta clasificación, excepto el test de Gordon, todos los demás tienen una consistencia interna aceptable o buena.

En esta investigación se encontró que el género no influyó significativamente en la viveza de imagen (VVIQ), lo que confirma la mayoría de los estudios precedentes (Ashton y White, 1980; Campos, 2014; Campos et al., 2002; Campos y Pérez, 1988b; McConkey y Nogrady, 1986). Tampoco influyó en el control de imagen (Gordon Test), lo que confirma los estudios de Campos et al. (2004) y de Pérez-Fabello y Campos (2004). Finalmente, el género tampoco influyó en la habilidad de imagen espacial (MASMI), lo que confirma los estudios de Campos (2009, 2013), Linn y Peterson (1985), Reilly y Neumann (2013), y Voyer et al. (1995).

Cuando se analizó la influencia del país en la viveza de imagen, se encontró que no influyó en la viveza de imagen, ni en la habilidad de imagen espacial, sin embargo, influyó significativamente en el control de imágenes. 
Este resultado corrobora los estudios precedentes, elaborados en otros países y comparando entre sí otros países en los que se observa que pueden existir diferencias entre países, dependiendo la medida utilizada y los países utilizados (Baranchok, 1995; Campos y Astorga, 1986a, 1986b, 1986c; Marsella y Quijano, 1974). Lamentablemente no hemos encontrado estudios precedentes españoles e italianos, ni con nuestras medidas de imagen, ni con otras medidas. Se necesitan nuevos estudios que hagan unos análisis comparativos entre países con distintos tipos de imagen, ya que, como hemos visto, pueden variar de unos países a otros.

\section{Referencias}

Ashton, R., \& White, K. D. (1980). Sex differences in imagery vividness: An artifact of the test. British Journal of Psychology, 71, 35-38. https://doi.org/10.1111/j.2044-8295.1980.tb02726.x

Baranchok, J. S. (1995). The linguistic and statistical equivalence of Spanish and English versions of Betts Questionnaire Upon Mental Imagery. Tesis Doctoral no publicada. Recuperada de https://tu-ir.tdl.org/ handle/2346/61170

Campos, A. (Ed.) (1998a). Imágenes mentales. Santiago de Compostela: Servicio de Publicaciones de la Universidad de Santiago de Compostela.

Campos, A. (1998b). A measure of visual imaging capacity: A preliminary study. Perceptual and Motor Skills, 87, 1012-1014. https://doi.org/10.2466/pms.1998.87.3.1012

Campos, A. (2009). Spatial imagery: A new measure of the visualization factor. Imagination, Cognition and Personality, 29, 31-39. https://doi.org/10.2190/IC.29.1.c

Campos, A. (2012). Measure of the ability to rotate mental images. Psicothema, 24, 431-434. https://doi.org/ $10.1037 / \mathrm{t} 14408-000$

Campos, A. (2013). Reliability and percentiles of a measure of spatial imagery. Imagination, Cognition and Personality, 32, 427-431. https://doi.org/10.2190/IC.32.e.f

Campos, A. (2014). Gender differences in imagery. Personality and Individual Differences, 59, 107-111. https://doi.org/10.1016/j.paid.2013.12.010

Campos, A., \& Astorga, V. M. (1986a). Spanish, North American, and Canadian ratings of imagery values of words. Perceptual and Motor Skills, 63, 889-890. https://doi.org/10.2466/pms.1986.63.2.889

Campos, A., \& Astorga, V. M. (1986b). Evocación de imaxes en suxeitos españois e canadienses: Unha análise comparativa. En M. Pérez (Ed.). Eidos de intervención en psicoloxía (pp. 243-246). Santiago de Compostela: Colexio Oficial de Psicólogos.

Campos, A., \& Astorga, V. M. (1986c). Evocación de imaxes en suxeitos españois e nordeamericanos: Unha análise comparativa. En M. Pérez (Ed.). Eidos de intervención en psicoloxía (pp. 251-258). Santiago de Compostela: Colexio Oficial de Psicólogos.

Campos, A., González, M. A., \& Amor, A. (2002). The Spanish version of the Vividness of Visual Imagery Questionnaire: Factor structure and internal consistency reliability. Psychological Reports, 90, 503-506. http://doi.org/10.2466/PR0.90.2.503-506

Campos, A., \& Pérez, M. J. (1988a). Vividness of Movement Imagery Questionnaire: Relations with other measures of mental imagery. Perceptual and Motor Skills, 67, 607-610. https://doi.org/10.2466/ pms.1988.67.2.607

Campos, A., \& Pérez, M. J. (1988b). Visual Elaboration Scale as measure of imagery. Perceptual and Motor Skills, 66, 411-414. https://doi.org/10.2466/pms.1988.66.2.411

Campos, A., Pérez-Fabello, M.J., \& Gómez-Juncal, R. (2004). Gender and age differences in measured and selfperceived imaging capacity. Personality and Individual Differences, 37, 1383-1389. http://dx.doi.org/10.1016/ j.paid.2004.01.008

Campos, A., \& Sueiro, E. (1993). Sex and age differences in visual imagery vividness. Journal of Mental Imagery, 17(3-4), 91-94. 
George, D., \& Mallery, P. (2003). SPSS for windows step by step: A simple guide and reference. 11.0 update. Boston: Allyn \& Bacon.

Horowitz, M. J. (1983). Image formation and psychoterapy. New York: Jason Aronson.

Linn, M. C., \& Peterson, A. C. (1985). Emergence and characterization of sex differences in spatial ability: A meta-analysis. Child Development, 56, 1479-1498. https://doi.org/10.2307/1130467

Marks, D. F. (1973). Visual imagery differences in the recall of pictures. British Journal of Psychology, 64, 1724. http://dx.doi.org/10.1111/j.2044-8295.1973.tb01322.x

Marks, D. F. (1995). New directions for mental imagery research. Journal of Mental Imagery, 19, 135-149.

Marsella, A. J., \& Quijano, W. Y. (1974). A comparison of vividness of mental imagery across different sensory modalities in Filipinos and Caucasian-Americans. Journal of Cross-Cultural Psychology, 5, 451-464. https://doi.org/10.1177/002202217400500406

McConkey, K. M., \& Nogrady, H. (1986). Visual Elaboration Scale: Analysis of individual and group version. Journal of Mental Imagery, 10, 37-46.

McKelvie, S. J. (1986). Effects of format of the vividness of visual imagery questionnaire on content validity, split-half reliability, and the role of memory in test-retest reliability. British Journal of Psychology, 77, 229236. https://doi.org/10.1111/j.2044-8295.1986.tb01997.x

Paivio, A., Yuille, J. C., \& Madigan, S. (1968). Concreteness, imagery, and meaningfulness values for 925 nouns. Journal of Experimental Psychology Monograph Supplement, 76, 1-25. http://dx.doi.org/10.1037/h0025327

Pérez-Fabello, M. J., \& Campos, A. (2004). Factor structure and internal consistency of the Spanish version of the Gordon Test of Visual Imagery Control. Psychological Reports, 94, 761-766. https://doi.org/10.2466/ PR0.94.3.761-766

Reilly, D., \& Neumann, D. L. (2013). Gender-role differences in spatial ability: A metaanalytic review. Sex Roles, 68, 521-535. https://doi.org/10.1007/s11199-013-0269-0

Richardson, A. (1969). Mental imagery. New York: Springer.

Richardson, A. (1977). The meaning and measurement of memory imagery. British Journal of Psychology, 68, 29-43. https://doi.org/10.1111/j.2044-8295.1977.tb01556.x

Sheehan, P. W. (1967). A shortened form of Betts' questionnaire upon mental imagery. Journal of Clinical Psychology, 23, 386-389. http://doi.org/fcfk5f

Varela, R. (2018). Las imágenes de la invención. Manuscrito no publicado. Santiago de Compostela: Consejo Superior de Investigaciones Científicas.

Voyer, D., Voyer, S., \& Bryden, M. P. (1995). Magnitude of sex differences in spatial abilities: A meta-analysis and consideration of critical variables. Psychological Bulletin, 117, 250-270. https://doi.org/10.1037/00332909.117.2.250

Walker, H. J. (1970). Imagery ratings for 338 nouns. Behavior Research Methods \& Instrumentation, 2, 165-167. http://doi.org/10.3758/BF03209293

White, K. D., Asthon, R., \& Brown, M. D. (1977). The measurement of imagery vividness: Normative data and their relationship to sex, age, and modality differences. British Journal of Psychology, 68, 203-211. https://doi.org/10.1111/j.2044-8295.1977.tb01576.x

Fecha de recepción: 15 de marzo de 2019.

Fecha de revisión: 28 de mayo de 2019.

Fecha de aceptación: 31 de mayo de 2019.

Fecha de publicación: 1 de julio de 2019. 